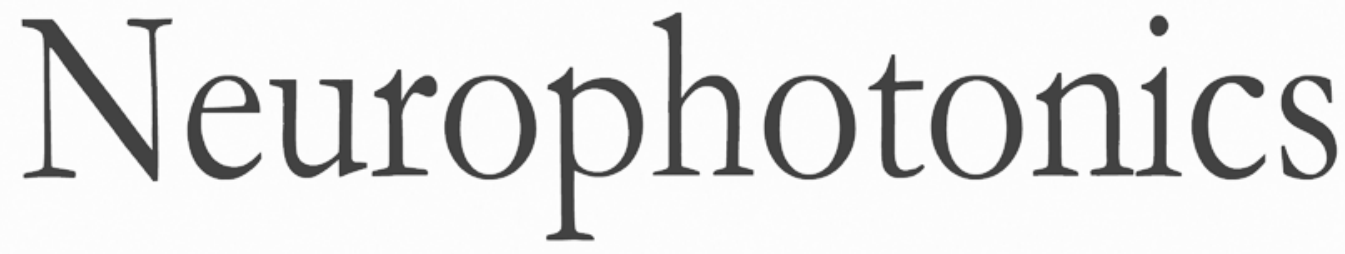

\title{
Abnormal dynamic functional connectivity and brain states in Alzheimer's diseases: functional near-infrared spectroscopy study
}

\author{
Haijing Niu \\ Zhaojun Zhu \\ Mengjing Wang \\ Xuanyu Li \\ Zhen Yuan \\ Yu Sun \\ Ying Han
}




\title{
Abnormal dynamic functional connectivity and brain states in Alzheimer's diseases: functional near-infrared spectroscopy study
}

\author{
Haijing Niu, ${ }^{a}$ Zhaojun Zhu, ${ }^{a, \dagger}$ Mengjing Wang, ${ }^{\text {a, } \dagger}$ Xuanyu Li, ${ }^{b}$ Zhen Yuan, ${ }^{c}$ Yu Sun,,${ }^{b, \star}$ and Ying Han ${ }^{b, d, e, f, *}$ \\ ${ }^{a}$ Beijing Normal University, State Key Laboratory of Cognitive Neuroscience and Learning and IDG/McGovern Institute for Brain Research, \\ Beijing, China \\ ${ }^{b}$ Xuan Wu Hospital of Capital Medical University, Department of Neurology, Beijing, China \\ 'University of Macau, Faculty of Health Sciences, Macao, China \\ 'Beijing Institute for Brain Disorders, Center of Alzheimer's Disease, Beijing, China \\ ${ }^{\mathrm{e} B e i j i n g}$ Institute of Geriatrics, Beijing, China \\ ${ }^{\mathrm{f}}$ National Clinical Research Center for Geriatric Disorders, Beijing, China
}

\begin{abstract}
Communication within the brain is highly dynamic. Alzheimer's disease (AD) exhibits dynamic progression corresponding to a decline in memory and cognition. However, little is known of whether brain dynamics are disrupted in $\mathrm{AD}$ and its prodromal stage, mild cognitive impairment (MCl). For our study, we acquired high sampling rate functional near-infrared spectroscopy imaging data at rest from the entire cortex of 23 patients with $\mathrm{AD}$ dementia, 25 patients with amnestic mild cognitive impairment $(\mathrm{aMCl})$, and 30 age-matched healthy controls (HCs). Sliding-window correlation and k-means clustering analyses were used to construct dynamic functional connectivity (FC) maps for each participant. We discovered that the brain's dynamic FC variability strength ( $Q$ ) significantly increased in both aMCl and $\mathrm{AD}$ group as compared to $\mathrm{HCs}$. Using the $Q$ value as a measurement, the classification performance exhibited a good power in differentiating aMCI [area under the curve (AUC = $82.5 \%)$ ] or $A D(A U C=86.4 \%$ ) from HCs. Furthermore, we identified two abnormal brain FC states in the $\mathrm{AD}$ group, of which the occurrence frequency $(F)$ exhibited a significant decrease for the first-level FC state (state 1) and a significant increase for the second-level FC state (state 2). We also found that the abnormal $F$ in these two states significantly correlated with the cognitive impairment in patients. These findings provide the first evidence to demonstrate the disruptions of dynamic brain connectivity in aMCI and $\mathrm{AD}$ and extend the traditional static (i.e., time-averaged) FC findings in the disease (i.e., disconnection syndrome) and thus provide insights into understanding the pathophysiological mechanisms occurring in aMCl and AD. ( $)$ The Authors. Published by SPIE under a Creative Commons Attribution 4.0 Unported License. Distribution or reproduction of this work in whole or in part requires full attribution of the original publication, including its DOI. [DOI: 10.1117/1.NPh.6.2.025010]
\end{abstract}

Keywords: resting state; functional connectivity; sliding-window; k-means clustering; brain network; Alzheimer's disease.

Paper 18077R received Dec. 19, 2018; accepted for publication May 2, 2019; published online Jun. 4, 2019.

\section{Introduction}

One of the most common neurodegenerative diseases, Alzheimer's disease $(\mathrm{AD})$ is characterized as a progressive decline in memory and other brain cognitive functions, likely caused by impaired structural integrity ${ }^{1,2}$ or abnormal functional connectivity (FC) among the nodes of interconnected brain regions or circuits. ${ }^{3-5}$ In addition, amnestic mild cognitive impairment (aMCI) is recognized as a transition state in the $\mathrm{AD}$ continuum, which exhibits a confirmed high risk (10\% to $15 \%)$ of progressing to $\mathrm{AD}{ }^{6}$ Interestingly, accumulating evidence also shows that $\mathrm{AD} / \mathrm{aMCI}$ is a disconnection syndrome. ${ }^{7-9}$

To date, FC for identifying the cognitive impairment in aMCI/AD has been widely performed in a time-averaged way, assuming that FC networks are spatiotemporally invariant (i.e., static) during task or at rest. However, constructed static networks might miss the dynamic features of brain activation or connectivity. Interestingly, recent advances in the analysis approach for dynamic FC have opened an avenue for improving

*Address all correspondence to Yu Sun, E-mail: 13810728286@126.com; Ying Han, E-mail: hanying@xwh.ccmu.edu.cn

†These authors contributed equally to this work. characterization of associated intrinsic brain activity. ${ }^{10-15}$ The time-varying or dynamic FC has been depicted by the temporal variability of $\mathrm{FC}$ at each connection, ${ }^{16-18}$ in which the transition dynamics between major FC patterns, known as FC states, show to be stable for a short period of time and reproducible across time and subjects. ${ }^{19}$ More importantly, recent research using these features of dynamic FC to inspect alterations in brain dynamics through both normal development ${ }^{20,21}$ and diseased progression, ${ }^{22,23}$ suggests that the temporal variability of FC is possibly related to changes in cognitive states. ${ }^{24}$ However, it is yet unclear whether the alterations also emerge in the time-varying properties of $\mathrm{FC}$ of $\mathrm{AD} / \mathrm{aMCI}$ and whether the time-varying properties of $\mathrm{FC}$ can serve as a neuromarker of AD/aMCI.

As a result, it is hypothesized in this study that the timevarying properties of FC should exhibit significant differences between AD/aMCI patients and healthy controls (HCs). To test this hypothesis, functional near-infrared spectroscopy (fNIRS) recordings were performed to inspect the spontaneous neural activity in 23 patients with $\mathrm{AD}$ dementia, 25 patients with aMCI, and $30 \mathrm{HCs}$. fNIRS is an emerging neuroimaging technique that can be used in a portable, comfortable, and quiet way, with few body constraints. fNIRS relies on the hemodynamic variations in oxygenated $(\mathrm{HbO})$, deoxygenated $(\mathrm{HbR})$, and total 
hemoglobin $(\mathrm{HbT})$ to infer brain activation and networks. The fNIRS imaging provides a relatively higher temporal sampling rate $(50 \mathrm{~Hz}$ in the current study) than traditional fMRI scanning, which facilitates elaborate characterization of dynamic brain connectivity in patients. Furthermore, the fNIRS technique is an ideal imaging tool for elderly participants including $\mathrm{AD}$ patients, who may have cognitive impairment and difficulties performing the tests. As such, we adopted fNIRS imaging data and combined sliding time window correlation and k-means clustering analysis to characterize brain dynamic FC and brain connectivity states for the three groups. Specifically, we focused on exploring the alterations in both brain FC dynamics and brain FC states in patients with aMCI and AD, as well as their possible association with clinical or behavioral variables.

\section{Materials and Methods}

\subsection{Participants}

From May 2016 to August 2017, 23 AD dementia patients, 25 aMCI patients, and 30 sex-, age-, and education-matched HCs participated in this study. All participants were right-handed Han Chinese with normal or corrected-to-normal version, who provided written informed consent for inclusion prior to the study. All clinical tests were approved by the Ethics Committee of the Beijing Normal (Beijing, China) and were then carried out as a standardized clinical evaluation, including a medical history interview and a battery of neuropsychological tests. The neuropsychological tests consisted of the Chinese version of the minimental state examination (MMSE) ${ }^{25}$ the Beijing version of Montreal cognitive assessment (MoCA), ${ }^{26}$ clinical dementia rating (CDR), the auditory verbal learning test (AVLT), ${ }^{27}$ Hachinski ischemic scale (HIS), Hamilton depression rating scale, ${ }^{28}$ the center for Epidemiologic Studies depression scale, ${ }^{29}$ and activities of daily living scale. Patients with aMCI and AD dementia were recruited from the memory clinic of the Neurology Department, Xuan Wu Hospital, Capital Medical University (Beijing, China), whereas the HCs were recruited from the local community by advertisements. The diagnoses of aMCI and $\mathrm{AD}$ dementia were determined by the consensus of two experienced neurologists according to the published criteria from Petersen et al..$^{30}$ and National Institute on AgingAlzheimer's Association, ${ }^{31}$ respectively. For details, the inclusion criteria for aMCI were as follows: (1) a memory complaint confirmed by an informant; (2) cognitive decline in a single domain or multiple domains; abnormal objective cognitive impairment documented by scores falling 1.5 SD below the age and education matched-specific norms on standard neuropsychological tests; (3) CDR score of 0.5; and (4) being free from dementia according to the Diagnostic and Statistical Manual of Mental Disorders, fourth edition, revised. The inclusion criteria for HCs were: (1) no complaint of memory or other cognitive impairment, (2) CDR score is 0, and (3) no severe visual or auditory impairment. All the participants were excluded if they demonstrated: (1) a clear history of stroke; (2) depression (Hamilton depression rating scale score $\geq 24$ points); (3) other nervous system diseases that can cause cognitive impairment (such as brain tumors, Parkinson's disease, encephalitis, or epilepsy); (4) traumatic brain injury; (5) other systemic diseases that can cause cognitive impairment, such as thyroid dysfunction, severe anemia, syphilis, and HIV; and (6) histories of psychosis or congenital mental growth retardation.

\section{2 fNIRS Data Acquisition}

The fNIRS scanning was conducted in a dimly lit room in Xuan Wu hospital. All participants were instructed to sit in a comfortable chair and remain motionless with their eyes closed [Fig. 1(a)], to avoid sleeping, and not to think about anything. The fNIRS data were acquired using a CW6 optical imaging system (CW6, Techen Co., Massachusetts) with a 46-channel array of optodes. This array consisted of 12 light emitters and 24 optical detectors [Figs. 1(b) and 1(c)], each of which detected the NIR light of its neighboring/surrounding emitters. The sources and detectors were alternately placed on each participant's right and left hemispheres with an interoptode distance of $3.2 \mathrm{~cm}$. The bottom column of the probe array was placed along the inion reference point according to the international $10 / 20$ system $^{32,33}$ so that the midpoint of the lower edge of the array was placed directly above the inion. The probe positioning was examined and adjusted to ensure consistency of the positions among the participants. Resting state was defined as no specific cognitive task during the fMRI scanning. The restingstate fNIRS data acquisition lasted $\sim 11$ min for each individual participant.

fNIRS data from individual channels were acquired at two different wavelengths $(690$ and $830 \mathrm{~nm})$ with a sampling rate of $50 \mathrm{~Hz}$. The signal quality was evaluated using signal-to-noise ratio, which was calculated as the ratio between mean signal and stand deviation of the raw signal on 690 and $830 \mathrm{~nm}$, respectively. Generally, the signal was acceptable for the signal-tonoise ratio larger than $2 \mathrm{~Hz}$. Changes in oxygenated $(\mathrm{HbO})$, deoxygenated ( $\mathrm{HbR})$, and total hemoglobin $(\mathrm{HbT})$ signals were generated by using the modified Beer-Lambert law. ${ }^{34}$ The $\mathrm{HbO}$ signal was primarily analyzed for the present study due to its high signal-to-noise in the correlation with regional cerebral blood flow. ${ }^{35}$ The HbR results were also analyzed and presented in the supplement as a complement.

For each individual's $\mathrm{HbO}$ dataset, a temporal independent component analysis (ICA) was conducted to remove typical motion-induced artifacts and systematic physiological noise. ${ }^{36-38}$ Specifically, these noise components were identified according to the components' temporal profiles, spatial maps, and power spectra. A component would be considered noise if it met one of the following conditions: $:^{37}$ (1) the corresponding temporal profile included sudden jumps, slowly varied $U$ or inverted U-shaped spike, or numerous intercurrent quick spikes (e.g., motion artifacts); (2) the dominant frequency of power spectra of the component was outside the range of 0.01 to $0.1 \mathrm{~Hz}$; and (3) the spatial map of the component presented a global and spatially dispersive pattern (e.g., physiological interference). Once the noise components were identified, the concentration signal was subsequently reconstructed with these particular components eliminated from the original hemoglobin time course by replacing zero in the corresponding column of mixing matrix. ${ }^{38}$ After the ICA procedure, a bandpass filter from 0.01 to $0.1 \mathrm{~Hz}$ was implemented to the denoised hemoglobin signals and then 10-min recordings (i.e., 30,000 time points) from the continuous time courses were extracted for later analysis.

\subsection{Dynamic FC Computation}

Figure 1(d) illustrates the dynamic FC analysis steps. Similar to the approach adopted by Allen et al. ${ }^{19}$ and in our previous study, ${ }^{39}$ a sliding window-based correlation approach was first utilized to generate a series of dynamic FC maps. Specifically, 
(a)

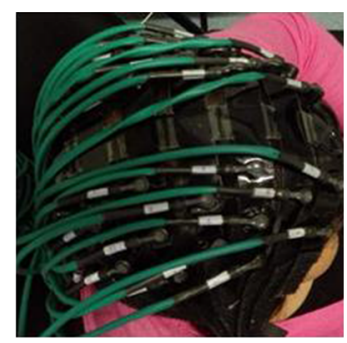

(d)

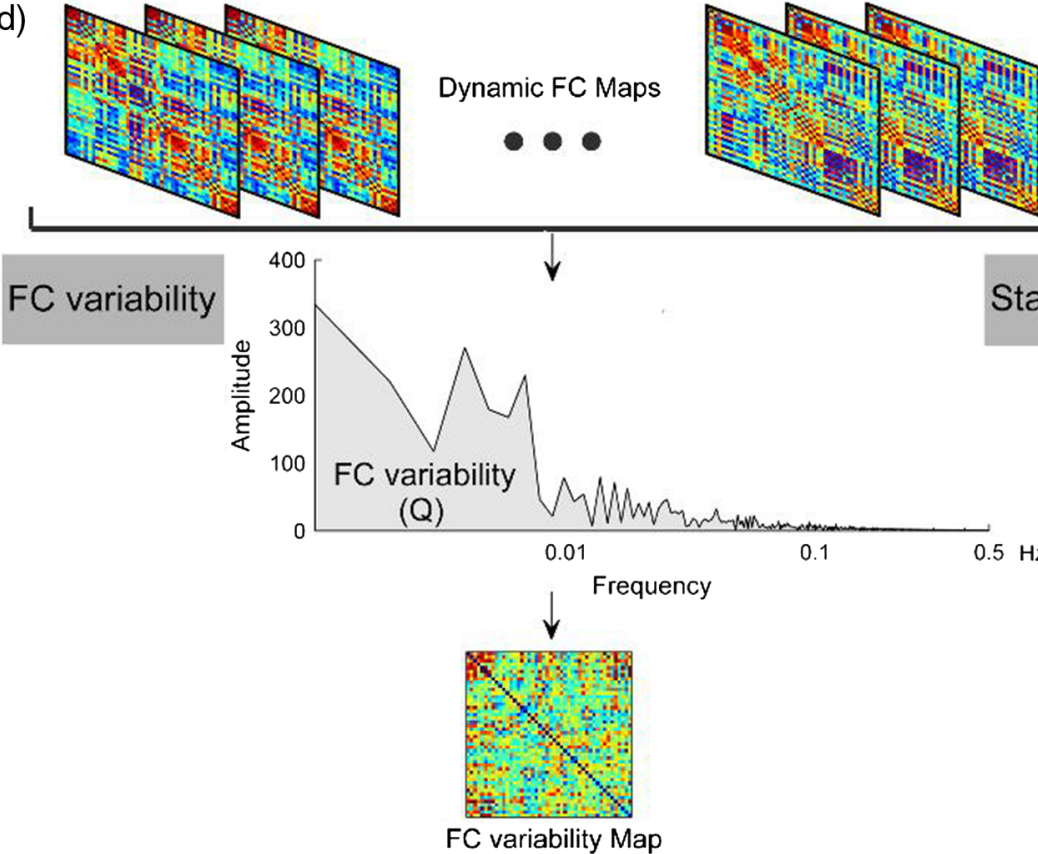

(b)

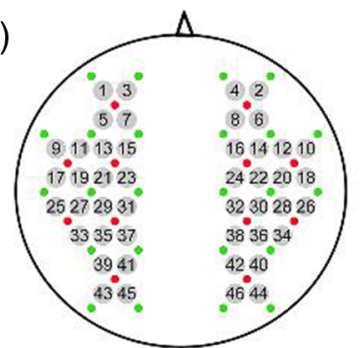

(c)

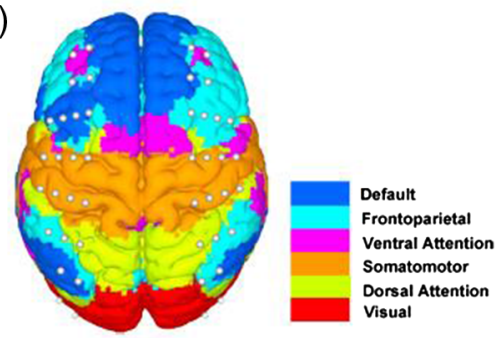

Dynamic FC Maps

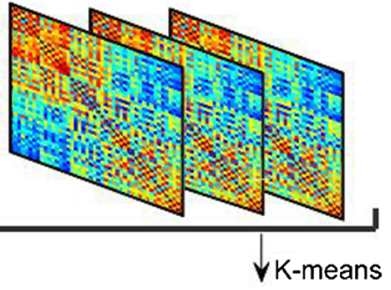

State Detection

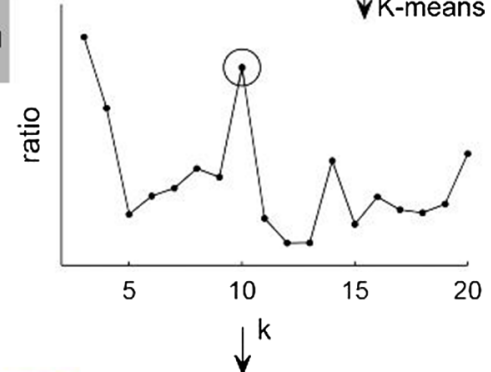

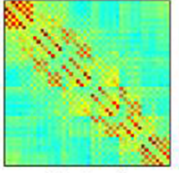

State 1

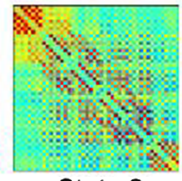

State 2

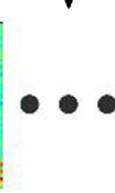

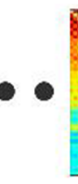

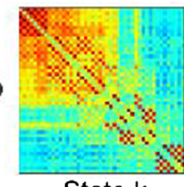

State $\mathrm{k}$

Fig. 1 (a) Photograph of fNIRS data collection from a participant. (b) The arrangement of the 46 measurement channels across the whole head. The red and green solid circles represent sources and detectors, respectively. The digits represent the positions of the measurement channels. (c) Anatomical position of each measurement channel. (d) Illustration of dynamic FC analysis steps. The spectrum power of the dynamic FC time series for each channel was first extracted. The AUC of the power spectrum across the low frequency $(<0.1 \mathrm{~Hz})$ band was then used as a dynamic FC variability index to mark time-varying characteristics of dynamic FC. K-means clustering was utilized to identify brain FC states.

for time series (i.e., $10 \mathrm{~min}$ ) on all measurement channels, 60-s time windows were selected and then shifted in an increment of $1 \mathrm{~s}$ along the entire time length. The FC within each time window was quantitatively calculated between any pair of brain regions using the Pearson correlation strategy. The 10-min measurement duration and a 60 -s time window brought about 541 sliding time windows and thus constructed 541 dynamic FC maps. To quantify the whole-cortex FC fluctuation across a series of sliding time windows, we also calculated the FC variability index $(Q)^{19,39}$ on each participant. Specifically, we first of all calculated the spectrum power of the dynamic FC time series (with Fourier analysis), ${ }^{19}$ and then the area under the curve (AUC) of the power spectrum across the low frequency $(<0.1 \mathrm{~Hz})$ band was quantified as the $\mathrm{FC}$ variability index, $Q$, to mark time-varying characteristics of dynamic FC. A larger $Q$ value denoted more variable FC, whereas a smaller $Q$ value represented less variable FC. Of note, the $Q$ index takes into account the information of power and amplitude in the frequency domain by filtering the potential fluctuating noises contained in the high frequencies $(>0.1 \mathrm{~Hz})$, which is slightly different from a simple measure of standard deviation. ${ }^{40} \mathrm{We}$ point out that the static FC was also calculated based on total signal length with Pearson correlation analysis.

\subsection{Dynamic FC Differences between the Various Groups}

To localize the specific pairs of regions in which the dynamic FC was altered in patient groups, we introduced a network-based statistic (NBS) approach ${ }^{41}$ to compare group difference in FC variability $Q$. The identified alternation in the value of index $Q$ was shown in the brain cortex according to their different spatial connectivity patterns: homotopic connectivity, long intrahemispheric connectivity, short intrahemispheric connectivity, and heterotopic connectivity. The details for comparison can be found in Sec. 2.6. The receiver operating characteristic (ROC) curve approach was further used to evaluate whether the $Q$ metric might serve as a biomarker for diagnosing patients from HCs. ROC is a graphical plot that illustrates the performance of a binary classifier, which was created by plotting the sensitivity against the 1 -specificity at various thresholds. 


\subsection{Detection and Characterization of FC States}

To detect brief FC patterns (i.e., FC states), we applied a $\mathrm{k}$-means clustering algorithm to the windowed correlation matrices concatenated across all participants. The clustering procedure was the same as the one used by Allen et al. ${ }^{19}$ The number of clusters $(k)$ was determined by using the elbow criterion of the cluster validity index that was denoted as the ratio between between-cluster distances to the within-cluster distance. For each cluster, we separately calculated the averaged FC map to represent the identified brain FC state. We also calculated the mean of the standard deviation among these FC maps, which characterized the FC variance within each brain state (i.e., cluster).

To demonstrate the difference in FC states between the patients and $\mathrm{HC}$ groups, we further introduced the metric of occurrence frequency, $F$, to characterize the occurrence of each FC state during dynamic brain activity. The metric $F$ was defined as: $F=N_{\mathrm{i}} / N_{\mathrm{t}}$, in which $N_{i}$ is the number of windows classified into a particular state and $N_{t}$ is the total number of the sliding time windows for a given time series. As such, for each participant, the occurrence frequency $(F)$ in each FC state could be quantified. For each FC state, the mean $F$ value was used as a statistical index for the comparison between the patients and heathy controls.

\subsection{Statistical Analysis}

\subsubsection{Group differences in clinical characteristics}

To test the group differences in age, gender, years of education, and neuropsychological scores, we analyzed the data with separate one-way analysis of variance (ANOVA). Posthoc pairwise comparisons were then performed using $t$ tests. The gender data were analyzed using the $\chi^{2}$ test.

\subsubsection{Group differences in dynamic FC variability}

To localize the specific pairs of regions in which the dynamic FC variability was altered in the aMCI or AD patients, we used a NBS approach. ${ }^{41}$ The NBS is a nonparametric test that controls for familywise error rate (FWER) at the network level using permutation testing. Due to the stringent FWER correction at the network level, no additional multiple comparison correction was performed for NBS analyses. Currently, the NBS approach has been used to identify abnormal brain connectivity circuitry in depression, ${ }^{42,43}$ Schizophrenia, ${ }^{44} \mathrm{AD},{ }^{4}$ and attention-deficit/ hyperactivity disorder. ${ }^{45}$

In this study, the NBS analysis primarily consisted of several steps. First, we performed two-sample one-tailed $t$-tests in an element-by-element manner on the temporal variability of all functional connections between aMCI and $\mathrm{HC}$ groups. Then, a primary cluster-defining threshold (e.g., $p<0.05$ and $p<0.005$ in this study, respectively) was used to identify suprathreshold connections, in which the connected components and the number of connections included in these components were determined. Third, to estimate the significance for each component, a null distribution of connected component size was derived empirically using a nonparametric permutation approach (10,000 permutations). For each permutation, all subjects were reallocated randomly into two groups, and twosample one-tailed $t$-tests were conducted for the same set of connections mentioned above. The same primary threshold ( $p<$ 0.05 and $p<0.005$, respectively) was then used to generate suprathreshold connections within which the maximal connected component size was recorded. Finally, for a connected component of size $N$ found in the right grouping of HCs and patients, the corrected $p$-value was determined by calculating the proportion of the 10,000 permutations for which the maximal connected component was larger than $N$. A similar analysis for group difference between the $\mathrm{AD}$ and $\mathrm{HC}$ groups was also conducted. It is noted that before the permutation tests, the effects of age, gender, and years of education were removed by a regression analysis.

\subsubsection{Group differences in FC state expressions}

To test the group differences in FC state properties under a specific state, comparisons were performed among the three groups using univariate ANCOVAs. Posthoc pairwise comparisons were then performed using a general linear model. The effects of age, gender, and years of education were adjusted for all of these analyses. A value of $p<0.05$ was considered significant.

\subsubsection{Relationships between clinical variables and FC variability or FC states}

We conducted analysis of multiple linear regressions in the participant groups to inspect the relationship between clinical variables and brain index. Figures 3(c) and 3(d) show the scatter plots with the association of clinical variables (MMSE score) and the values $Q$ of FC variability. Figure 7 shows the scatter plots with the association of clinical variables (MMSE score, AVLT-recognition, AVLT-delayed recall, and MoCA) and the $F$ values of FC state occurrence.

\section{Results}

\subsection{Demographic and Clinical Characteristics}

The demographic data are shown in Table 1 . There were no significant differences in age $[F(2,75)=1.891 ; p=0.16]$, gender $(p=0.40)$, or years of education $[F(2,75)=1.07 ; p=$ $0.35]$ among the three groups. However, the patient group (aMCI and $\mathrm{AD}$ ) had significantly lower scores on the MMSE $[F(2,75)=51.51, p<0.001]$, MoCA $[F(2,75)=53.80, p<$ $0.001]$, AVLT-immediate recall $[F(2,75)=58.34, p<0.001]$, AVLT delayed recall $[F(2,75)=118.52, p<0.001]$, and AVLT-recognition $[F(2,75)=65.26, p<0.001]$ than the HC group (Table 1).

\subsection{Visualization of Dynamic FC Variability}

Using sliding window correlation analysis and Fourier analysis, we obtained quantitative FC variability $Q$ at each connection for the HCs, aMCI, and AD groups, respectively [Fig. 2(a)]. Visually, the FC variability was obviously increased in participants during the progression of $\mathrm{HCs}$ to $\mathrm{aMCI}$ to $\mathrm{AD}$, with most of the connections showing larger $Q$ in $\mathrm{AD}$ and smaller $Q$ in HCs [Fig. 2(b)]. Quantitatively, the results also support the observation that averaged $Q$ values across all 1035 (i.e., $46 \times$ $45 / 2$ ) connections were $4.2 \pm 0.16$ for $\mathrm{HCs}, 4.5 \pm 0.18$ for aMCI, and $4.7 \pm 0.17$ for AD. One possible explanation for the larger $Q$ in $\mathrm{AD}$ is that $\mathrm{AD}$ patients could primarily stay in an aberrant brain state while that brain state could show larger brain FC fluctuations. The static FC was also calculated for the HCs, aMCI, and AD groups [Fig. 2(c)]. Visually, the static FC 
Table 1 Demographics and clinical characters of the participants.

\begin{tabular}{lcccc}
\hline Characteristics & HC & aMCl & AD & $p$ Value \\
\hline$N(\mathrm{M} / \mathrm{F})$ & $30(11 / 19)$ & $25(13 / 12)$ & $23(8 / 15)$ & $0.40^{\mathrm{a}}$ \\
Age (years) & $67.63 \pm 9.02$ & $71.04 \pm 8.13$ & $72.09 \pm 9.32$ & $0.16^{\mathrm{b}}$ \\
Education (years) & $11.73 \pm 5.34$ & $10.56 \pm 5.12$ & $9.70 \pm 4.70$ & $0.35^{\mathrm{b}}$ \\
MMSE & $28.23 \pm 3.07$ & $23.48 \pm 4.81$ & $15.52 \pm 5.74$ & $<0.01^{\mathrm{b}}$ \\
MoCA & $25.53 \pm 3.81$ & $18.92 \pm 5.39$ & $10.65 \pm 5.23$ & $<0.01^{\mathrm{b}}$ \\
AVLT_I & $9.73 \pm 2.26$ & $6 \pm 2.53$ & $3.52 \pm 2.04$ & $<0.01^{\mathrm{b}}$ \\
AVLT_D & $11.53 \pm 2.36$ & $3.70 \pm 3.27$ & $1.36 \pm 1.97$ & $<.0 .01^{\mathrm{b}}$ \\
AVLT_R & $13.07 \pm 1.72$ & $7.61 \pm 3.33$ & $4.36 \pm 2.97$ & $<0.01^{\mathrm{b}}$ \\
\hline
\end{tabular}

Note: Data are presented as the range of minimum-maximum (mean $\pm \mathrm{SD}$ ).

${ }^{a}$ The $p$ value was obtained using a two-tailed Pearson chi-squared test.

' The $p$ value was obtained using one-way analysis of variance tests.

(a)

$\boldsymbol{Q}$

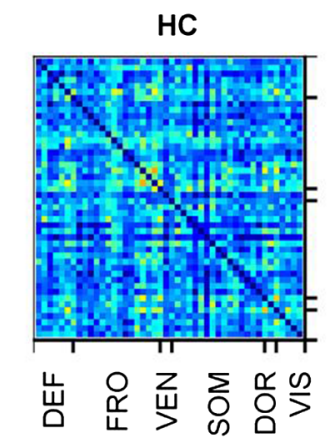

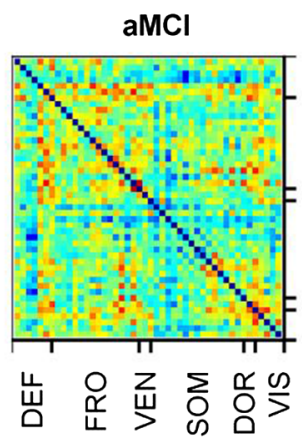

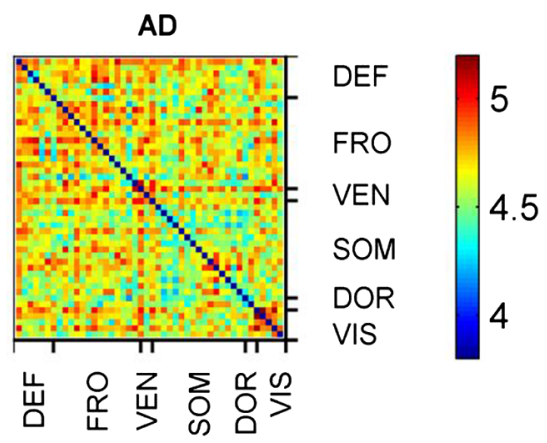

(b)

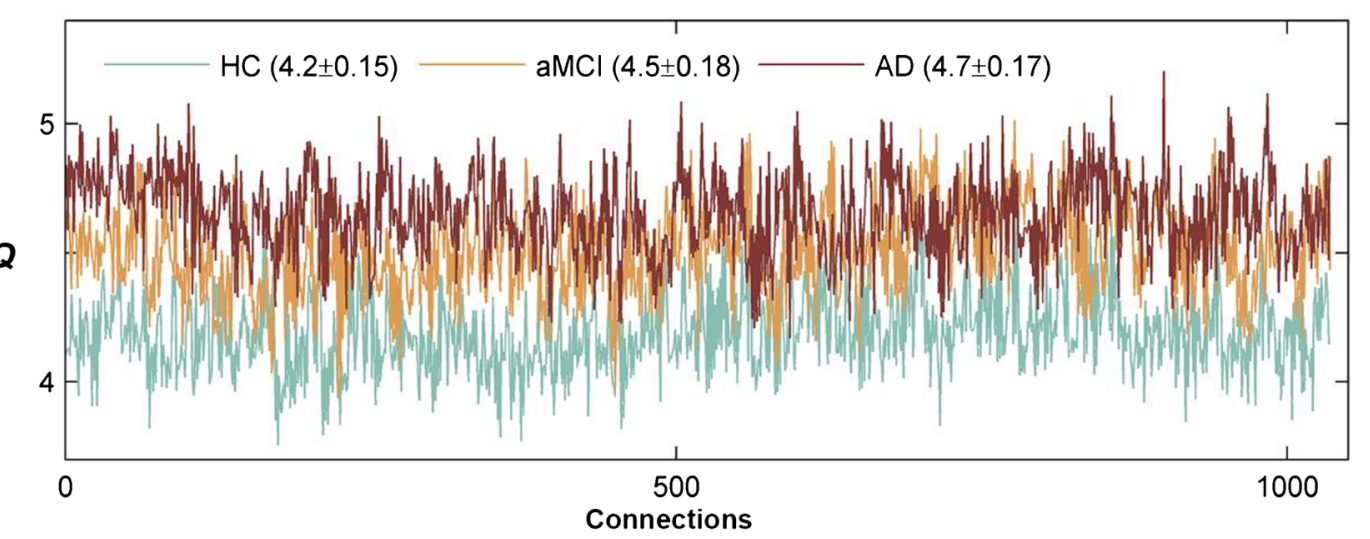

(c)

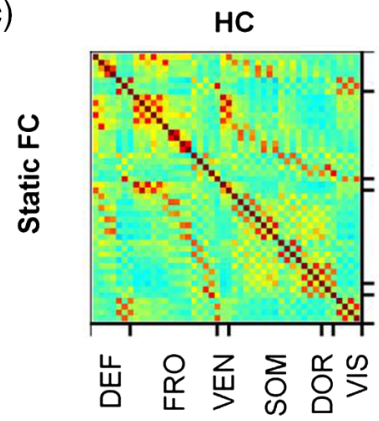

aMCI

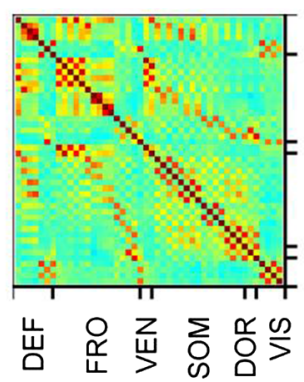

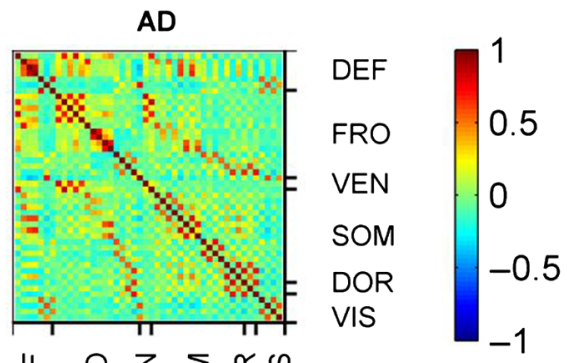

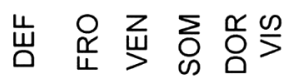

4.5

4

Fig. 2 (a) Maps of $\mathrm{FC}$ variability for $\mathrm{HC}$, aMCl, and $\mathrm{AD}$ groups, respectively. The $Q$ values represent the variability of dynamic $\mathrm{FC}$ over time. (b) One-dimensional representation of the $\mathrm{FC}$ variability $(Q)$ for these three groups. (c) Maps of static $\mathrm{FC}$ for $\mathrm{HC}, \mathrm{aMCl}$, and $\mathrm{AD}$ groups, respectively. The values represent the static functional connectivity strength. 
maps showed similar spatial patterns across the $\mathrm{HCs}$, aMCI, and AD groups.

\subsection{Disrupted Regional FC Dynamics in aMCl and $A D$}

We used the NBS method to identify the disrupted connected components in patients (Fig. 3). Under the cluster defining threshold of $p<0.05$, networks with 307 and 548 connections were revealed to experience significant changes in dynamic FC variability in the aMCI and AD groups, respectively. Using a more rigorous threshold of $p<0.005$, the networks were reduced to 34 and 178 significant connections for aMCI and $\mathrm{AD}$, respectively.
For the aMCI group, the significantly changed connections (using the threshold of $p<0.005$ ) tended to be relatively long distance and were concentrated in regions including the prefrontal and parietal cortexes [Fig. 3(a)]. We further considered the roles of these regional structures in the context of the functional network organization derived from the $\mathrm{HC}$ group. We found that the targeted connections in the aMCI group belong mainly to the default mode network, and the internetwork connections were mainly between the default mode network and the frontalparietal network. For the AD group, a single connected network consisting of 178 edges linking widely distributed brain regions was altered [Fig. 3(b)]. The involved connections also belonged mainly to long connections and were concentrated in regions including the prefrontal, parietal, and visual cortexes. We further (a)

$\mathrm{aMCl}>\mathrm{HC}$

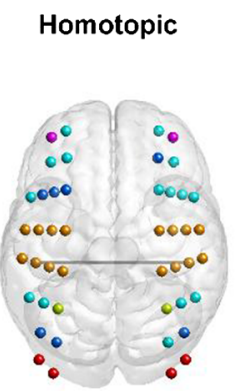

(b)

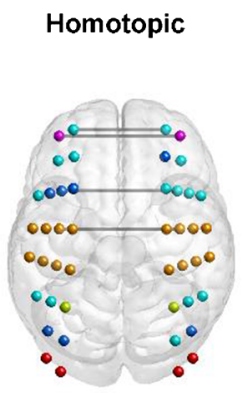

(c)

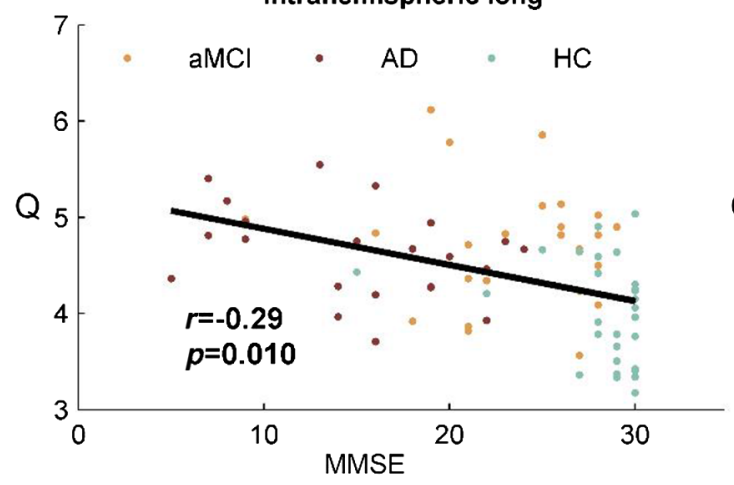

Intrahemispheric Short
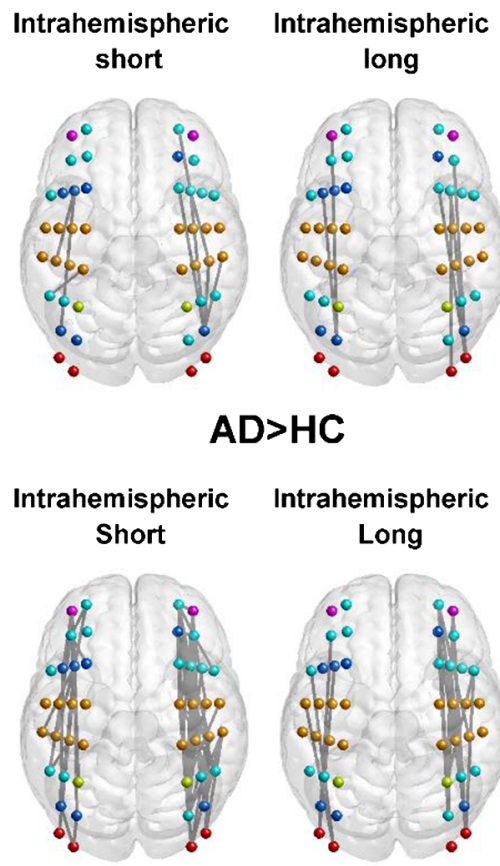

$A D>H C$

Intrahemispheric Long

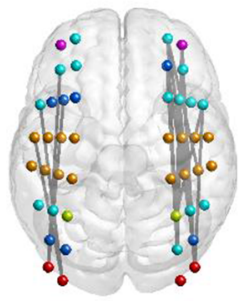

Heterotopic

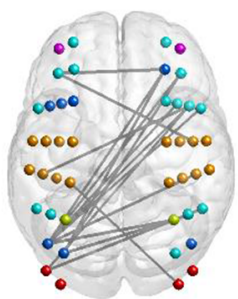

Heterotopic

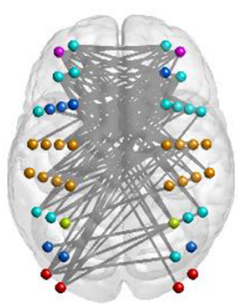

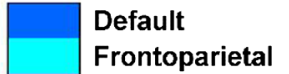

Ventral Attention

Somatomotor

Dorsal Attention

Visual

Fig. 3 The brain regions with significantly increased $Q$ values in patients with (a) aMCl and (b) $A D$. The significant connections were categorized into four connectivity subgroups, i.e., homotopic, intrahemispheric long and short, and heterotopic connections. The fNIRS measurement channels with different colors showed different regions of functional networks. (c) The relationship between FC variability $Q$ and MMSE scores in the significant intrahemispheric long connections located in (a). (d) The relationship between FC variability $Q$ and MMSE scores in the significant intrahemispheric long connections located in panel (b).

(d)

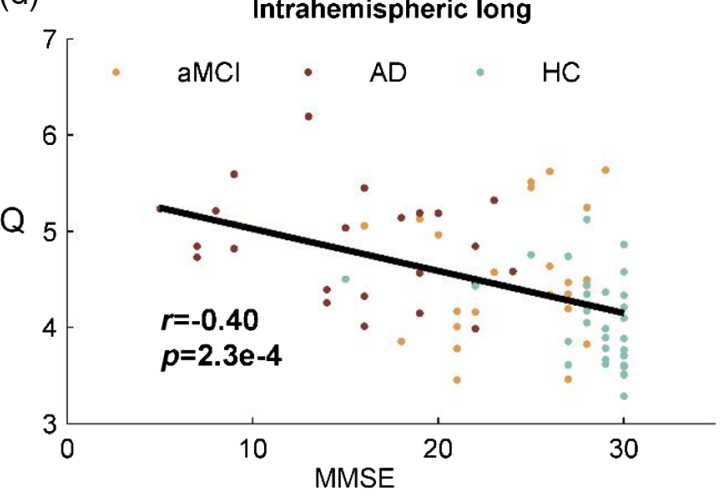


found that the targeted connections in the $\mathrm{AD}$ group belong mainly to the within default mode network, the frontal-parietal network, and the internetwork connections that linked the functional regions in the somatosensory, visual, and frontalparietal networks. Notably, all the significant connections found in the aMCI group were also found in the AD group at network level.

All of the connections exhibited increased dynamic FC variability in the patients compared with the controls. No significant differences were found with respect to connected components between the patient groups of aMCI and $\mathrm{AD}$ patients. For the static FC, no significant differences were found between the patients (i.e., aMCI or AD) and HCs. For dynamic FC, the $Q$ were significantly negatively related to the MMSE scores in the intrahemispheric long connections [Figs. 3(c) and 3(d)], irrespective of the long connections from aMCI [Fig. 3(a)] or AD [Fig. 3(b)]. More severely, impaired patients tended to have increased $Q$ in the long intrahemispheric connections that were abnormal in patients.

(a)

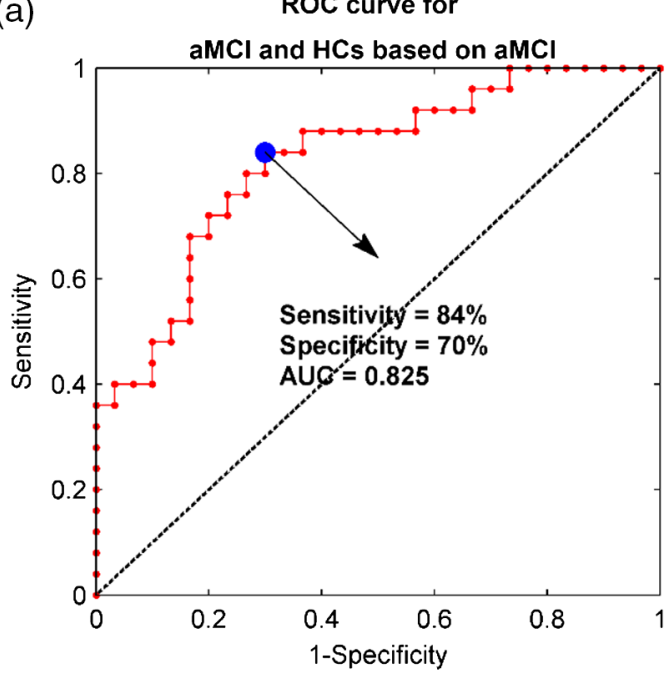

(c)

ROC curve for

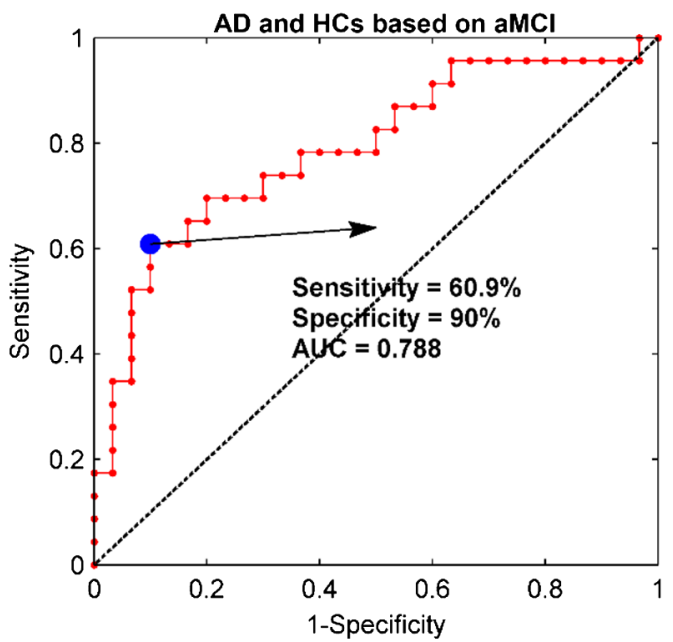

\subsection{Sensitivity and Specificity of Dynamic FC Variability in Differentiating Patients from HCs}

Figure 4 showed the sensitivity and specificity of the index $Q$ in differentiating patients from HCs. The measurement was the mean $Q$ across significant connections between aMCI and HCs [Figs. 4(a) and 4(c)] and between AD and HCs [Figs. 4(b) and 4(d)], respectively. Overall, the classification exhibited a satisfactory power in differentiating patients from HCs, no matter which measurement was used. Specifically, to differentiate aMCI patients from $\mathrm{HCs}$, the classification performance was better as using the measurement from aMCI group [Fig. 4(a): sensitivity $=84 \%$, specificity $=70 \%$, and AUC $=82.5 \%]$ than that from $\mathrm{AD}$ group [Fig. 4(b): sensitivity $=84 \%$, specificity $=$ $50 \%$, and $\mathrm{AUC}=71.2 \%$ ]. Likewise, to differentiate $\mathrm{AD}$ patients from $\mathrm{HCs}$, the classification performance was better as using the measurement from AD group [Fig. 4(d): sensitivity = $82.6 \%$, specificity $=76.7 \%$, and $\mathrm{AUC}=86.4 \%$ ] than that from aMCI group [Fig. 4(c): sensitivity $=60.9 \%$, specificity $=90 \%$, and $\mathrm{AUC}=78.8 \%$ ]. These results demonstrate that the index

(b)

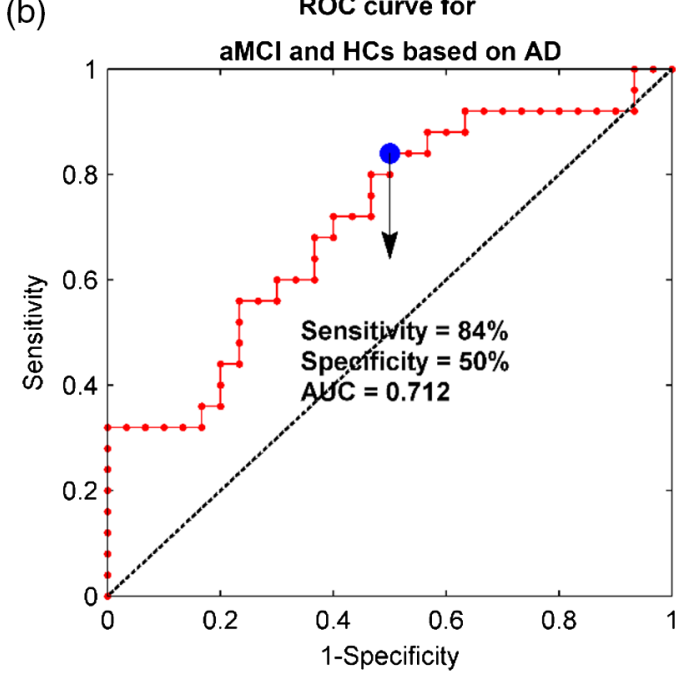

(d)

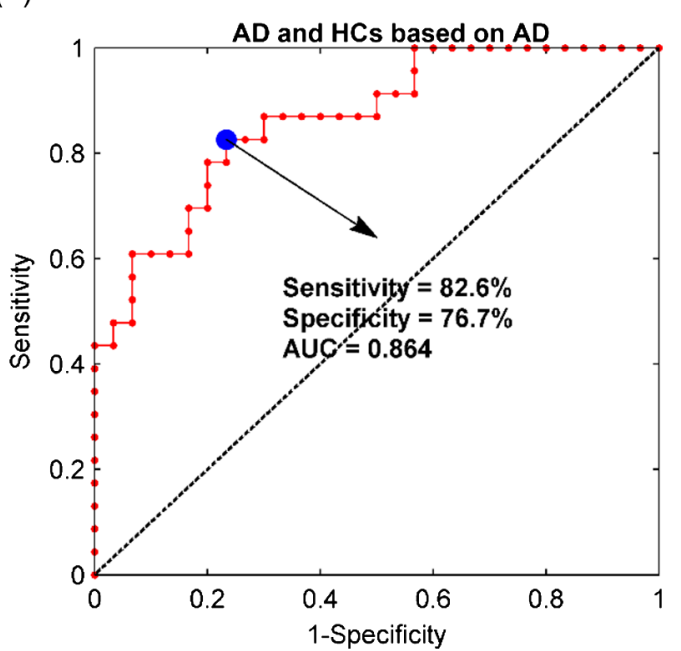

Fig. 4 The patients-controls classification. (a) and (b) The ROC curves of average $Q$ for aMCl and $\mathrm{HCs}$, in which the average $Q$ was calculated from the significant connections between $\mathrm{aMCl}$ and $\mathrm{HCs}(\mathrm{a})$, and between $A D$ and HCs (b), respectively. (c) and (d) The ROC curves of average $Q$ for AD and HCs, in which the average $Q$ was calculated from those significant connections between $\mathrm{aMCl}$ and $\mathrm{HCs}(\mathrm{a})$, and between $A D$ and $H C s(d)$, respectively. 


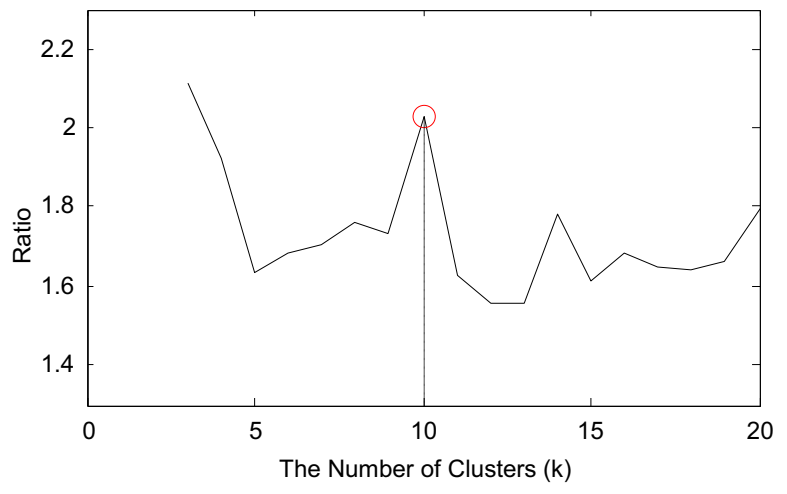

Fig. 5 The plot of cluster number versus ratio of between-cluster and within-cluster distance.

$Q$ could be used as a biomarker for the diagnosis of patients with aMCI and AD.

\subsection{FC States across Patients and HCs}

Figure 5 shows the plot of cluster number versus ratio of between-cluster and within-cluster distance. Ten FC states were observed across multiple participants. We extracted four FC states that dynamically reoccurred over time and across participants in both patients and HCs [Fig. 6(a)]. The participants expressed an average of 2.13 (SD, 0.97), 2.08 (SD, 0.86), and 2.26 (SD, 0.69) states for $\mathrm{HCs}$, aMCI, and $\mathrm{AD}$, respectively. The number of states expressed had no significant difference between participant groups.

Of the four FC states that were observable across participant groups, state 1 accounts for $\sim 61 \%$ of all windows, followed by state 2 for $23 \%$, state 3 for $13 \%$, and state 4 for $3 \%$ of all windows. The FC pattern in state 1 closely resembled the static FC [Fig. 6(b), correlation coefficient $r=0.96$ ], with strong positive correlations along the diagonal (i.e., within functional networks) [Fig. 6(a)], consistent with previous dynamic findings, ${ }^{46}$ which represented a steady FC. For state 2, we found that the FC in the default mode network, frontal-parietal network, and visual network was increased, although it was decreased in the attention and somatosensory networks compared to state 1 . The patterns in both the whole-brain FC and the within subnetwork connectivity for state 2 were very similar to those in state 1 , but the variance of FC patterns within state 2 (which was quantified as the standard deviation across all FC patterns within each state) was larger than that within state 1 [Fig. 6(c)]. For states 3 and 4, the FC patterns were less observed and also differed markedly from these patterns of states 1 and 2 . Notably, the patterns showed quite uniform connectivity between different measurement channels, which might reflect signals from global and noncortical components. As such, the states 3 and 4 are not analyzed or discussed in the following sections.

\subsection{Group Differences in Occurrence Frequency $(F)$ of FC States}

Analysis of group differences in $F$ values exhibited significant group effects (all $p<0.05$ ) for state $1[F(2,72)=3.581, p=$ $0.033]$ and state $2[F(2,72)=8.532, p<0.001]$, respectively. Posthoc comparisons revealed significantly reduced $F$ values in state 1 and significantly increased $F$ values in state 2 for $\mathrm{AD}$ patients relative to both the controls and the aMCI patients (all $p<0.05$ ) (Fig. 7). However, no differences were found in the $F$ values between the aMCI and controls (all $p>0.05$ ) for these two states.

\subsection{Relationship between State Occurrence Frequency $(F)$ and Behavioral Performance}

The occurrence frequencies $F$ for state 1 and state 2 showed different linear relationships with the clinical variables of the

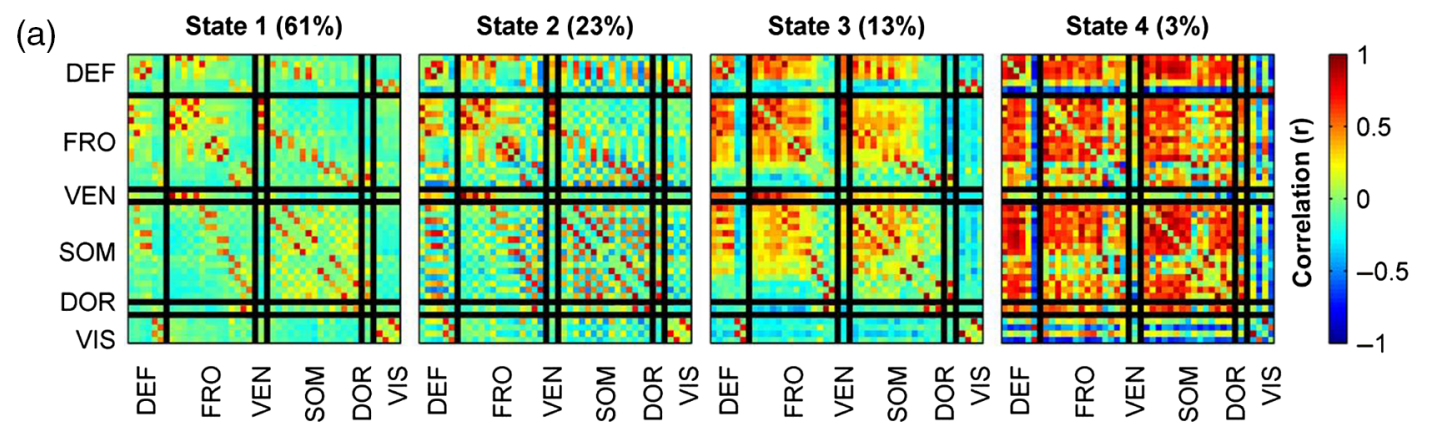

(b)

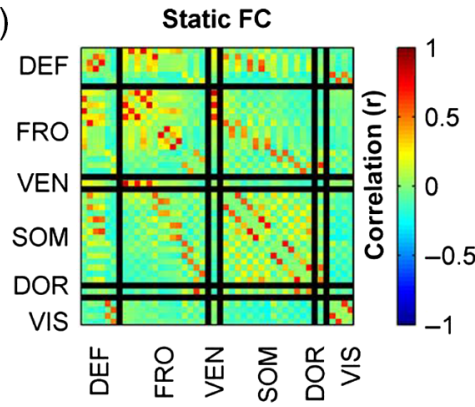

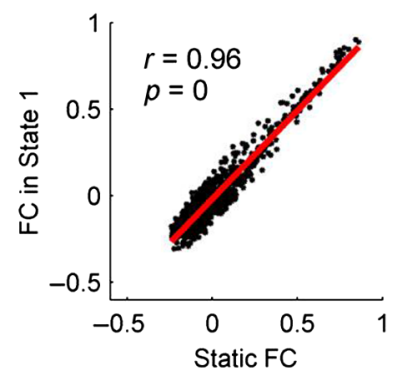

(c)

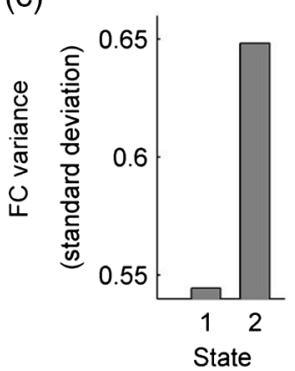

Fig. 6 (a) These four FC states were characterized over all the subjects. (b) The FC pattern in state 1 closely resembled the static FC with a correlation coefficient of $r=0.96$. (c) FC variance (standard deviation) within FC state 1 and state 2 . 


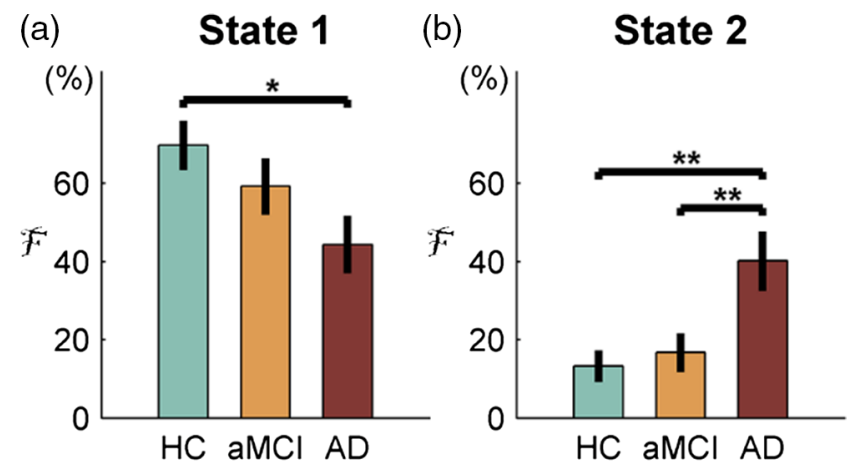

Fig. 7 Group differences of occurrence frequency $F$ in FC states 1 and 2 among $\mathrm{HCs}, \mathrm{aMCl}$, and $\mathrm{AD}$.

patients (Fig. 8). For state 1, the index $F$ showed a significant positive correlation with the MMSE $(r=0.40, p=0.0062)$ as well as the AVLT_recognition $(r=0.37, p=0.015)$ [Fig. 8(a)]. For state 2, the index $F$ showed a significant negative correlation with the MMSE $(r=0.39, p=0.0087)$, MoCA $(r=0.3$, $p=0.05)$, AVLT_delayed recall $(r=0.35, p=0.025)$, and the AVLT_recognition $(r=0.31, \quad p=0.046)$ [Fig. 8(b)], respectively.

\section{Discussion}

We applied high sampling rate fNIRS imaging data to investigate brain connectivity dynamics in patients with aMCI and AD. We found that, compared to HCs, the patient groups showed increased dynamic FC $(Q)$ and abnormal occurrence frequency (F) in specific brain connectivity states. These findings demonstrate that dynamic brain FC networks were disrupted in patients with aMCI and $\mathrm{AD}$, which provides an important complement to traditional time-averaged FC studies in aMCI/AD patients, which solely characterize disconnection syndrome on the brain connectivity strength.
The human brain is a complex network with time-varying FC and network organization. A large number of neuroimaging studies have revealed the dynamic characteristics of functional cerebral network in human brain. ${ }^{12,19,47,48}$ In this study, our results, for the first time assessed time-varying brain FC alternation in $\mathrm{aMCI}$ and $\mathrm{AD}$ patients. We showed that dynamic brain FC was significantly increased in the patient groups (Figs. 2 and 3). More importantly, our results revealed that the influence of aMCI/AD was preferentially on long-distance connections, e.g., involving in the regions of the default mode network and frontal-parietal network. The default mode network and frontal-parietal network were considered to be susceptible and vulnerable regions to AD pathology. ${ }^{49-52}$ As such, the abnormal fluctuation of dynamic brain FC in these regions could reflect dysregulation of network function in facilitating information integration across large-scale brain networks in the patients with aMCI/AD. Our findings are also consistent with previous neuroimaging studies from static brain connectivity analysis in AD. ${ }^{53-57}$ These combined results demonstrate that both brain connectivity strength and brain connectivity fluctuation were disrupted in $\mathrm{AD}$ patients, which consistently impacts efficient information integration between different functionally segregated brain regions.

Furthermore, our results also showed that, using a ROC analysis and the significant measurement separately from aMCI or $\mathrm{AD}$, the mean measurement values (i.e., the mean $Q$ ) can differentiate aMCI [Fig. 4(a)] or AD [Fig. 4(d)] patients from HCs with high sensitivity and specificity (Fig. 4), suggesting that the measurement of index $Q$ could be a potential marker for diagnosing aMCI or AD patients. By contrast, the static FC did not show significant changes between patients and HCs. These comparisons demonstrate that the metric $Q$ of dynamic FC could be much more sensitive compared to static FC in differentiating aMCI or AD patients from HCs. Such a reliable marker will have tremendous value as a tool for early detection of AD-related brain disease. (a)

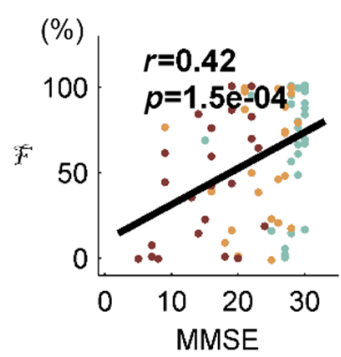

(b)

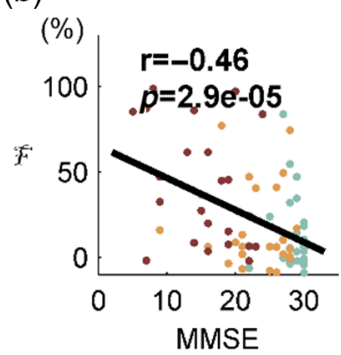

State 1

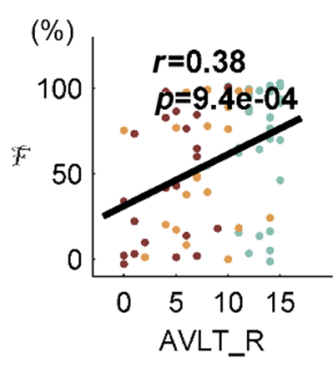

State 2

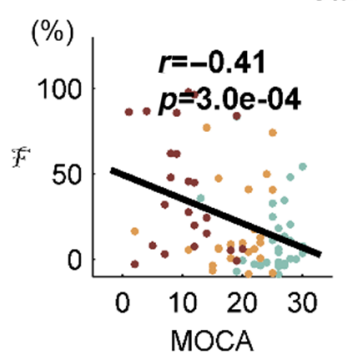

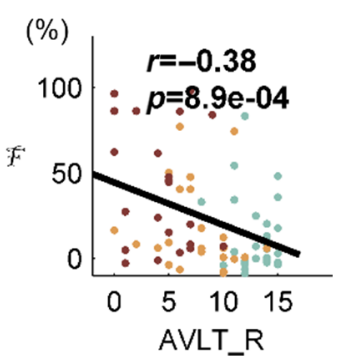

Fig. 8 The correlations between the occurrence frequency $F$ and clinical variables. (a) Plots showing the significant increases of the $F$ in state 1 with MMSE and AVLT_R scores. (b) Plots showing the significant decreases of the $F$ in state 2 with MMSE, MoCA, AVLT_D, and AVLT_R. 
The FC states are considered to be some short-term connectivity patterns; they are highly replicable and, in part, diverge strongly from stationary connectivity patterns. We identified aberrant brain FC states in aMCI/AD patients. Specifically, the emergency frequency $(F)$ in specific brain FC states significantly changed between patient groups and HCs. For example, AD patients exhibited a significantly decreased $\mathrm{F}$ in the first-level connectivity states (i.e., FC state 1). The FC state 1 represented the stationary connectivity-like pattern and emerged most frequently in dynamic brain activity. ${ }^{58,59}$ The state 1 accounted for $\sim 70 \%$ in the dynamic connectivity and the other connectivity states account for much less frequency (ranging between $7 \%$ and $15 \%)$. The decrease in $F$ of FC state 1 in AD patients suggested that an important and stable connectivity pattern was disrupted in AD patients during spontaneous neural activity.

Furthermore, we also found that the $F$ in the second-level connectivity states (i.e., FC state 2) was significantly increased in $\mathrm{AD}$ patients. The $\mathrm{FC}$ state 2 primarily characterized the connections in default mode network and frontal-parietal and visual networks, and it showed a less frequent emergency during dynamic brain activity in healthy individuals [Fig. 4(c)]. However, for $\mathrm{AD}$ patients, the increase of the $F$ in FC state 2 demonstrated much larger brain connectivity instability in the patient groups. Similar to our findings, Damaraju et al. ${ }^{60}$ also point out that specific connectivity states were altered in schizophrenic patients. As such, we speculate that the abnormal change in brain FC states could lead to disrupted functional integration throughout the brain in $\mathrm{AD}$ patients, which may further account for cognitive deficits in these patients.

It is also noted that the altered $F$ in brain FC states correlated specifically with abnormal cognitive performance in patients. For example, the $F$ in FC states 1 and 2 significantly correlated with the typical cognitive scores, e.g., MMSE and the AVLT_ recognition, respectively [Figs. 8(a) and 8(b)]. These findings demonstrated that the disruption of occurrence frequency in specific connectivity states seriously correlated with the alternation of brain cognition performance in $\mathrm{AD}$ patients. It also suggested that the aberrant FC state is likely to underlie the decline in cognitive and memory functions. Overall, our study demonstrated that both brain connectivity fluctuation and brain connectivity states were disrupted in $\mathrm{AD}$ patients, which extends the current understanding about human brain connectome in this disease, and shows the importance of evaluating dynamic changes of brain connectivity in AD.

Several issues need to be further addressed. First, we adopted resting-state fNIRS imaging data to characterize dynamic FC in $\mathrm{AD} / \mathrm{aMCI}$. One of the most important advantages to use fNIRS for dynamic FC analysis is the capability of long-term data collection. However, in this study, we collected only 10-min data length on each participant. Future study will exploit longer fNIRS scan duration (e.g., several hours) to improve estimates of FC variability by allowing patterns of connectivity to reoccur several more times. Second, the present study was crosssectional, in which individual variations existed and may impact the current main findings. Considering the convenience of using fNIRS to conduct repeatable data collection, future studies with longitudinal fNIRS imaging are required to further validate the findings observed in this study. Third, a potential drawback ${ }^{61}$ of using fNIRS imaging to assess functional network organization in $\mathrm{AD}$ or aMCI patients is that, unlike fMRI, fNIRS imaging is limited to the superficial cerebral cortex (i.e., $\sim 1-2 \mathrm{~cm}$ into the brain) and cannot access deep cortical structures (e.g., the insula or operculum) or subcortical brain structures (e.g., the striatum or thalamus). Fortunately, a number of abnormal brain regions are relatively near the cortical surface, including large portions of the anterior and posterior regions of the default mode network cortex, which are frequently highlighted in fMRI studies. Finally, it is important to note that significant differences in FC state features between aMCI and HCs were not found in our study. This result could be because aMCI is a transitional and long period in the progression of $\mathrm{AD}$ and that different individuals at different stages are probably too heterogeneous to present uniform FC state patterns. On the other hand, the results could be affected by the small sample size. As such, it would be important to recruit more participants to validate our current conclusions in the future.

\section{Conclusion}

The present study provided a methodological framework for applying dynamic FC analysis to AD/aMCI patients. The results revealed that both brain connectivity fluctuation and brain connectivity states were disrupted in AD patients, which extends the current understanding about human brain connectome in this disease and provides insights into understanding the pathophysiological mechanisms occurring in aMCI and AD.

\section{Disclosures}

The authors have declared that no conflict of interest exists.

\section{Acknowledgments}

This study is supported by the Natural Science Foundation of China (Grant Nos. 81761148026, 81571755, 81430037, 61633018, and 81471731), the National Key Research and Development Program of China (Grant No. 2016YFC1306300), Beijing Municipal government (Grant No. PXM2018, 026283_000002), Beijing Nature Science Foundation (Grant Nos. 7161009 and 7132147), and Beijing Municipal Science \& Technology Commission (Grant No. Z161100002616020).

\section{References}

1. I. H. Choo et al., "Relationship of fibrillar beta-amyloid burden with age-at-onset in Alzheimer's disease," Alzheimer's Dementia J. Alzheimers Dis. Assoc. 6(4), S39-S40 (2010).

2. L. Zhuang et al., "White matter integrity in mild cognitive impairment: a tract-based spatial statistics study," Neurolmage 53(1), 16-25 (2010).

3. Y. He et al., "Neuronal networks in Alzheimer's disease," Neuroscientist 15(4), 333-350 (2009).

4. J. Wang et al., "Disrupted functional brain connectome in individuals at risk for Alzheimer's disease," Biol. Psychiatry 73(5), 472-481 (2013).

5. C. Sorg et al., "Selective changes of resting-state networks in individuals at risk for Alzheimer's disease," Proc. Natl. Acad. Sci. U. S. A. 104(47), 18760-18765 (2007).

6. R. C. Petersen et al., "Mild cognitive impairment: clinical characterization and outcome," Arch. Neurol. 56(3), 303-308 (1999).

7. M. Bozzali et al., "Regional grey matter loss and brain disconnection across Alzheimer disease evolution," Curr. Med. Chem. 18(16), 2452-2458 (2011)

8. X. Delbeuck, F. Collette, and M. Van der Linden, "Is Alzheimer's disease a disconnection syndrome?: evidence from a crossmodal audiovisual illusory experiment," Neuropsychologia 45(14), 3315-3323 (2007)

9. X. Delbeuck, M. Van der Linden, and F. Collette, "Alzheimer'disease as a disconnection syndrome?" Neuropsychol. Rev. 13(2), 79-92 (2003).

10. C. Chang and G. H. Glover, "Time-frequency dynamics of resting-state brain connectivity measured with fMRI," NeuroImage 50(1), 81-98 (2010). 
11. D. A. Handwerker et al., "Periodic changes in fMRI connectivity," NeuroImage 63(3), 1712-1719 (2012).

12. R. M. Hutchison et al., "Dynamic functional connectivity: promise, issues, and interpretations," NeuroImage 80, 360-378 (2013).

13. R. M. Hutchison et al., "Resting-state networks show dynamic functional connectivity in awake humans and anesthetized macaques," Hum. Brain Mapping 34(9), 2154-2177 (2013).

14. M. Breakspear, "Dynamic models of large-scale brain activity," Nat. Neurosci. 20(3), 340-352 (2017).

15. A. Avena-Koenigsberger, B. Misic, and O. Sporns, "Communication dynamics in complex brain networks," Nat. Rev. Neurosci. 19(1), 17-33 (2018).

16. A. Zalesky et al., "Time-resolved resting-state brain networks," Proc. Natl. Acad. Sci. U. S. A. 111(28), 10341-10346 (2014).

17. T. Matsui, T. Murakami, and K. Ohki, "Neuronal origin of the temporal dynamics of spontaneous BOLD activity correlation," Cereb. Cortex 29, 1496-1508 (2019).

18. T. O. Laumann et al., "On the stability of BOLD fMRI correlations," Cereb. Cortex 27(10), 4719-4732 (2017).

19. E. A. Allen et al., "Tracking whole-brain connectivity dynamics in the resting state," Cereb. Cortex 24(3), 663-676 (2014).

20. R. M. Hutchison and J. B. Morton, "Tracking the brain's functional coupling dynamics over development," J. Neurosci. 35(17), 6849-6859 (2015).

21. H. A. Marusak et al., "Dynamic functional connectivity of neurocognitive networks in children," Hum. Brain Mapping 38(1), 97-108 (2017).

22. J.-M. Yu et al., "The prognostic value of long-term visit-to-visit blood pressure variability on stroke in real-world practice: a dynamic cohort study in a large representative sample of Chinese hypertensive population," Int. J. Cardol. 177(3), 995-1000 (2014).

23. X. B. Chen et al., "Extraction of dynamic functional connectivity from brain grey matter and white matter for MCI classification," Hum. Brain Mapping 38(10), 5019-5034 (2017).

24. A. Kucyi and K. D. Davis, "Dynamic functional connectivity of the default mode network tracks daydreaming," Neurolmage 100, 471-480 (2014).

25. J. Jia et al., "The recommendation of diagnosis and treatment of cognitive impairment in Chinese elderly," Chin. J. Geriatr. 33(8), 817-825 (2014).

26. J. Lu et al., "Montreal cognitive assessment in detecting cognitive impairment in Chinese elderly individuals: a population-based study," J. Geriatr. Psychiatry Neurol. 24(4), 184-190 (2011).

27. Q. Guo, "Norm of auditory verbal learning test in the normal aged in Chinese community," Chin. J. Clin. Psychol. 15(2), 132-135 (2007).

28. M. Hamilton, "A rating scale for depression," J. Neurol. Neurosurg. Psychiatry 23(1), 56-62 (1960).

29. E. Dozeman et al., "The center for epidemiological studies depression scale (CES-D) is an adequate screening instrument for depressive and anxiety disorders in a very old population living in residential homes," Int. J. Geriatr. Psychiatry 26(3), 239-246 (2011).

30. R. C. Petersen et al., "Current concepts in mild cognitive impairment," Arch. Neurol. 58(12), 1985-1992 (2001).

31. G. M. McKhann et al., "The diagnosis of dementia due to Alzheimer's disease: recommendations from the National Institute on AgingAlzheimer's Association workgroups on diagnostic guidelines for Alzheimer's disease," Alzheimer's Dementia J. Alzheimer's Assoc. 7(3), 263-269 (2011).

32. V. Jurcak et al., "Virtual 10-20 measurement on MR images for intermodal linking of transcranial and tomographic neuroimaging methods," NeuroImage 26(4), 1184-1192 (2005).

33. V. Jurcak, D. Tsuzuki, and I. Dan, "10/20, 10/10, and 10/5 systems revisited: their validity as relative head-surface-based positioning systems," NeuroImage 34(4), 1600-1611 (2007).

34. M. Cope and D. T. Delpy, "System for long-term measurement of cerebral blood and tissue oxygenation on newborn infants by near infra-red transillumination," Med. Biol. Eng. Comput. 26(3), 289-294 (1988).

35. X. Cui, D. M. Bryant, and A. L. Reiss, "NIRS-based hyperscanning reveals increased interpersonal coherence in superior frontal cortex during cooperation," NeuroImage 59(3), 2430-2437 (2012).

36. S. Kohno et al., "Removal of the skin blood flow artifact in functional near-infrared spectroscopic imaging data through independent component analysis," J. Biomed. Opt. 12(6), 062111 (2007).
37. H. Niu et al., "Test-retest reliability of graph metrics in functional brain networks: a resting-state fNIRS study," PLoS One 8(9), e72425 (2013).

38. H. Zhang et al., "Functional connectivity as revealed by independent component analysis of resting-state fNIRS measurements," NeuroImage 51(3), 1150-1161 (2010).

39. $\mathrm{Z}$. Li et al., "Dynamic functional connectivity revealed by resting-state functional near-infrared spectroscopy," Biomed. Opt. Express 6(7), 2337-2352 (2015)

40. J. Liu et al., "Chronnectome fingerprinting: identifying individuals and predicting higher cognitive functions using dynamic brain connectivity patterns," Hum. Brain Mapping 39(2), 902-915 (2018).

41. A. Zalesky, A. Fornito, and E. T. Bullmore, "Network-based statistic: identifying differences in brain networks," NeuroImage 53(4), 11971207 (2010).

42. F. Bai et al., "Topologically convergent and divergent structural connectivity patterns between patients with remitted geriatric depression and amnestic mild cognitive impairment," J. Neurosci. 32(12), 4307-4318 (2012).

43. J. R. Zhang et al., "Disrupted brain connectivity networks in drugnaive, first-episode major depressive disorder," Biol. Psychiatry 70(4), 334-342 (2011)

44. A. Zalesky et al., "Disrupted axonal fiber connectivity in schizophrenia," Biol. Psychiatry 69(1), 80-89 (2011).

45. Q. J. Cao et al., "Probabilistic diffusion tractography and graph theory analysis reveal abnormal white matter structural connectivity networks in drug-naive boys with attention deficit/hyperactivity disorder," J. Neurosci. 33(26), 10676-10687 (2013).

46. M. Fukushima et al., "Characterizing spatial patterns and flow dynamics in functional connectivity states and their changes across the human lifespan," Quant. Biol. (2015).

47. D. G. McLaren, R. A. Sperling, and A. Atri, "Flexible modulation of network connectivity related to cognition in Alzheimer's disease," NeuroImage 100, 544-557 (2014)

48. A. Patanaik et al., "Dynamic functional connectivity and its behavioral correlates beyond vigilance," NeuroImage 177, 1-10 (2018).

49. M. D. Greicius et al., "Default-mode network activity distinguishes Alzheimer's disease from healthy aging: evidence from functional MRI," Proc. Natl. Acad. Sci. U. S. A. 101(13), 4637-4642 (2004).

50. M. Pievani et al., "Functional network disruption in the degenerative dementias," Lancet Neurol. 10(9), 829-843 (2011).

51. M. W. Cole et al., "Multi-task connectivity reveals flexible hubs for adaptive task control," Nat. Neurosci. 16(9), 1348-1355 (2013).

52. H. J. Li et al., "Toward systems neuroscience in mild cognitive impairment and Alzheimer's disease: a meta-analysis of 75 fMRI studies," Hum. Brain Mapping 36(3), 1217-1232 (2015).

53. R. L. Buckner et al., "Cortical hubs revealed by intrinsic functional connectivity: mapping, assessment of stability, and relation to Alzheimer's disease," J. Neurosci. 29(6), 1860-1873 (2009).

54. Z. J. Dai et al., "Identifying and mapping connectivity patterns of brain network hubs in Alzheimer's disease," Cereb. Cortex 25(10), 3723-3742 (2015).

55. Y. Bar-Yam, Dynamics of Complex Systems, Springer, The Netherlands (2006).

56. E. J. Sanz-Arigita et al., "Loss of 'Small-World' networks in Alzheimer's disease: graph analysis of fMRI resting-state functional connectivity," PLoS One 5(11), e13788 (2010).

57. Y. Liu et al., "Impaired long distance functional connectivity and weighted network architecture in Alzheimer's disease," Cereb. Cortex 24(6), 1422-1435 (2014).

58. J. Cabral, M. L. Kringelbach, and G. Deco, "Exploring the network dynamics underlying brain activity during rest," Prog. Neurobiol. 114, 102-131 (2014)

59. Q. B. Yu et al., "Assessing dynamic brain graphs of time-varying connectivity in fMRI data: application to healthy controls and patients with schizophrenia," NeuroImage 107, 345-355 (2015).

60. E. Damaraju et al., "Dynamic functional connectivity analysis reveals transient states of dysconnectivity in schizophrenia," NeuroImage Clin. 5, 298-308 (2014).

61. H. Niu and Y. He, "Resting-state functional brain connectivity: lessons from functional near-infrared spectroscopy," Neuroscientist 20(2), 173-188 (2014). 
Haijing Niu received her MS and PhD degrees in optics from Tianjin University and Beijing Normal University, followed by postdoctoral training in biomedical optics at the University of Texas at Arlington. She is an associate professor of the State Key Lab of Cognitive Neuroscience and Learning at Beijing Normal University, China. She focuses on resting-state fNIRS (rs-fNIRS) imaging studies (e.g., using rs-fNIRS data to study the human brain connectome), the software development of the rs-fNIRS data analysis, and the corresponding applications in both healthy brain development and brain diseases.

Zhaojun Zhu received his bachelor's degree in biomedical engineering from Xi'an University of Electronic Science and Technology in 2014. He is a graduate of the State Key Laboratory of Cognitive Neuroscience and Learning \& IDG/McGovern Institute for Brain Research, Beijing Normal University. His research interests are using fNIRS combined with graph theory to explore mental diseases, such as Alzheimer's disease.

Mengjing Wang received a bachelor's degree in biomedical engineering from the Central South University in 2017. She is a postgraduate in State Key Laboratory of Cognitive Neuroscience and Learning \& IDG/McGovern Institute for Brain Research, Beijing Normal University. Her research interests are using fNIRS combined with graph theory to explore mental diseases, such as attention-deficit hyperactivity disorder and Alzheimer's disease.
Xuanyu $\mathbf{L i}$ is a medical doctoral candidate in the Department of Neurology, Xuanwu Hospital of Capital Medical University. His work focuses on clinical research of neuroimaging in SCD in preclinical Alzheimer's disease.

Zhen Yuan is an associate professor with faculty of Health Sciences at University of Macau (UM). Before joined UM, he had worked as an assistant professor with Arizona State University and a research assistant professor with University of Florida. He received his $\mathrm{PhD}$ in engineering from University of Science and Technology of China in 2002. His research interests are focused on biomedical optics, neuroimaging and neuroscience, and molecular imaging and cancer.

Yu Sun is a medical doctoral candidate in the Department of Neurology, Xuanwu Hospital of Capital Medical University. She received her MSc degree from Capital Medical University. Her work focuses on clinical research of structural and functional neuroimaging in SCD of Alzheimer's disease.

Ying Han is a professor of neurology, Xuanwu Hospital of Capital Medical University. She is the initiator of the Alliance for Preclinical Alzheimer's Disease of China, National Clinical Research Center for Geriatric Disorders. Her research interests are prevention, diagnosis, and intervention for the Alzheimer's disease continuum, especially the early diagnosis of subjective cognitive decline by multimodal magnetic resonance imaging techniques and follow-up study. 